\title{
Melanoma oral primario. Revisión de la literatura
} Primary oral melanoma. Literature review Melanoma oral primário. Revisão da literatura

doi http://dx.doi.org/10.35954/SM2019.38.2.4

\author{
Hugo Néstor Mondón Alvarez ${ }^{a}$ \\ https://orcid.org/0000-0003-1808-0515 \\ Natalia Fernanda Tancredi Cueto ${ }^{b}$ \\ https://orcid.org/0000-0002-1663-0950
}

(a) Departamento de Odontología, Hospital Central de las Fuerzas Armadas.

(b) Docente Grado 2. Facultad de Odontología, Universidad de la República.

\begin{abstract}
RESUMEN
El melanoma oral primario de la mucosa es una neoplasia agresiva rara y representa solo el $0.2-8 \%$ de todos los melanomas reportados. Es una neoplasia maligna de melanocitos que puede surgir de una lesión melanocítica benigna o de novo de los melanocitos dentro de la piel o mucosa normales. Se considera la neoplasia humana más mortal y biológicamente impredecible, con el peor pronóstico debido principalmente, al diagnóstico inicial tardío, a la dificultad de alcanzar una resección amplia y a la tendencia de esta neoplasia de producir metástasis a distancia vía hematógena en forma muy temprana. Se estima que el $36 \%$ de los pacientes con melanoma oral primario presentan compromiso ganglionar regional al momento del diagnóstico y el $85 \%$ de ellos desarrollan metástasis al hígado, pulmones, hueso o cerebro en un corto periodo de tiempo. Actualmente el tratamiento más efectivo para los Melanomas es la resección quirúrgica con amplios márgenes de seguridad, complementada con la disección de cuello para metástasis de ganglios linfáticos positivos y en forma coadyuvante en pacientes de alto riesgo.
\end{abstract}

PAlabRAS ClaVe: Diagnóstico Bucal; Diagnóstico Precoz; Manifestaciones Bucales; Melanoma; Melanocitos; Neoplasias; Neoplasias de la Boca.

\begin{abstract}
Primary oral mucosal melanoma is a rare aggressive neoplasm and accounts for only $0.2-8 \%$ of all reported melanomas. It is a malignant melanocyte neoplasm that may arise from a benign melanocytic lesion or de novo melanocyte lesion within normal skin or mucosa. It is considered the most deadly and biologically unpredictable human neoplasm, with the worst prognosis mainly due to the late initial diagnosis, the difficulty of reaching a wide resection and the tendency of this neoplasm to produce metastases at a distance via hematogenous in a very early form. It is estimated that $36 \%$ of patients with primary oral melanoma present regional lymph node involvement at the time of diagnosis and $85 \%$ of them develop metastases to the liver, lungs, bone or brain in a short period of time. Currently, the most effective treatment for Melanomas is surgical resection with wide safety margins, complemented with neck dissection for positive and adjuvant lymph node metastases in high-risk patients.

Primary oral mucosal melanoma is a rare aggressive neoplasm and accounts for only $0.2-8 \%$ of all reported melanomas. It is a malignant melanocyte neoplasm that may arise from a benign melanocytic lesion or de novo melanocyte lesion within normal skin or mucosa. It is considered the most deadly and biologically
\end{abstract}


unpredictable human neoplasm, with the worst prognosis mainly due to the late initial diagnosis, the difficulty of reaching a wide resection and the tendency of this neoplasm to produce metastases at a distance via hematogenous in a very early form. It is estimated that $36 \%$ of patients with primary oral melanoma present regional lymph node involvement at the time of diagnosis and $85 \%$ of them develop metastases to the liver, lungs, bone or brain in a short period of time. Currently, the most effective treatment for Melanomas is surgical resection with wide safety margins, complemented with neck dissection for positive and adjuvant lymph node metastases in high-risk patients.

KEY WORDS: Diagnosis, Oral; Early Diagnosis; Oral Manifestations; Melanoma; Melanocytes; Neoplasms; Mouth Neoplasms.

\section{RESUMO}

O melanoma primário da mucosa oral é uma neoplasia agressiva rara e representa apenas 0,2-8\% de todos os melanomas relatados. É uma neoplasia melanocítica maligna que pode surgir de uma lesão melanocítica benigna ou de novo melanócito na pele ou mucosa normal. É considerada a neoplasia humana mais letal e biologicamente imprevisível, com pior prognóstico, principalmente devido ao diagnóstico inicial tardio, à dificuldade de obtenção de ampla ressecção e à tendência dessa neoplasia a produzir metástases à distância, via hematogênica, de forma muito precoce. Estima-se que $36 \%$ dos pacientes com melanoma oral primário apresentam comprometimento linfonodal regional no momento do diagnóstico e $85 \%$ deles desenvolvem metástases para o fígado, pulmão, osso ou cérebro em um curto período de tempo. Atualmente, o tratamento mais eficaz para os melanomas é a ressecção cirúrgica com amplas margens de segurança, complementada com dissecção cervical para metástases linfonodais positivas e adjuvantes em pacientes de alto risco.

PAlaVRas ChaVe: Diagnóstico Bucal; Diagnóstico Precoce; Manifestações Bucais; Melanoma; Melanócitos; Neoplasias; Neoplasias Bucais.

\section{INTRODUCCIÓN}

Este manuscrito tiene como objetivo presentar una revisión de la literatura sobre el melanoma oral primario (MOP).

EI MOP es una neoplasia maligna poco frecuente derivada de los melanocitos presentes en la mucosa oral. Su etiología y patogenia no ha sido esclarecida aún. Es considerado como un cáncer agresivo, de mal pronóstico (1). La mayoría de los MOP tienen un comportamiento biológico agresivo y muestran una alta incidencia de metástasis, recurrencia y muerte (2).

Al momento del diagnóstico la mayoría de los MOP se presentan como lesiones nodulares con un crecimiento vertical e infiltrante, en contraste con los melanomas cutáneos que son diagnosticados con frecuencia durante la fase de crecimiento radial (3).

\section{DESARROLLO}

El melanoma oral primario (MOP) es una forma rara de melanoma $(4,5)$ con un comportamiento clínico-patológico agresivo y un pronóstico pobre $(3,6,7)$. Se origina a partir de la transformación maligna de los melanocitos de la mucosa oral $(2,5,8,9)$, células de origen neuroectodérmico que se encuentran en la capa basal y parabasal de la mucosa oral (2) y ocasionalmente en la submucosa (6). Los melanocitos constituyen una población celular escasa en comparación con las células escamosas en la conformación del epitelio, lo que explicaría la rara ocurrencia del MOP (9). Intervienen en la homeostasis de piel y mucosas tanto en condiciones fisiológicas como patológicas, a través de la producción de melanina, melatonina y serotonina (2). 
EL MOP representa aproximadamente el $1.6 \%$ de todas las neoplasias malignas de cabeza y cuello y el 0.2 al $8 \%$ de todos los melanomas del organismo humano $(1,5,6,9,10)$. La edad de presentación es muy variable, registrándose casos desde los 7 hasta los 95 años (2), siendo diagnosticados con mayor frecuencia entre la quinta y séptima década de la vida. La edad pico para el diagnóstico del MOP tiende a ser una o dos décadas después que los melanomas cutáneos (5). La mayor prevalencia se observa en afroamericanos, japoneses e indios asiáticos (2), siendo ligeramente más frecuente en hombres que en mujeres (10). Los MOPs a diferencia del melanoma cutáneo, se originan de novo, a partir de la mucosa bucal aparentemente sana $(4,6)$, en sitios que no están expuestos a la radiación solar (6); posee un comportamiento biológico más agresivo en comparación con a otros cánceres orales y con los melanomas cutáneos $(2,6)$, siendo por tanto imperativo la detección y diagnóstico temprano para un mejor pronóstico (10).

La etiología y la patogénesis del MOP no ha sido establecida aún, tampoco se han reconocido posibles factores de riesgo $(2,4,6,9,10)$.

EI MOP suele ser asintomático en sus estadios iniciales, lo que explica en parte el diagnóstico tardío de la mayoría de las lesiones. Entre los síntomas habituales asociados a estadios avanzados se denotan dificultades en el uso de prótesis, dolor en áreas de ulceración, sangrado, movilidad dental y parestesia (2).

Clínicamente el MOP se presenta como una mácula o nódulo, asintomático, de color marrón oscuro o negro, generalmente de forma asimétrica, de bordes irregulares, pudiendo presentar zonas ulceradas y sangrantes $(2,5,9)$. Con frecuencia en las proximidades a la lesión principal se observan lesiones satélites(1). En su avance comprometen estructuras vecinas y erosionan el hueso subyacente $(5,11)$.

Se describe una variedad de MOP extremadamente rara que carece de pigmento (2), denominada amelánica, que en su presentación clínica recuerda a un carcinoma oral de células escamosas $(4,9)$. EI MOP se localiza con mayor frecuencia en paladar duro y en segundo lugar en la encía maxilar $(2,3,6,8)$, en la literatura se registran casos en piso de boca, mucosa bucal y encía mandibular (10). Según las características clínicas, los MOP se pueden dividir en cinco tipos: nodular pigmentado, nodular no pigmentado, macular pigmentado, mixto pigmentado y mixto no pigmentado (1). El tipo nodular no pigmentado es un tumor nodular amelanótico que no tiene una fase de crecimiento radial, mientras que el tipo mixto no pigmentado es un tumor nodular amelanótico que está rodeado por una fase de crecimiento radial (2). Frente al examen clínico de un posible MOP se debe plantear diagnóstico diferencial con otras lesiones de origen melánico, como ser: mácula melanótica oral, melanosis asociada al tabaquismo, melanosis inducida por fármacos (como minociclina y drogas antipalúdicas), melanoacantoma, pigmentación asociada a procesos inflamatorios, nevus, tatuaje de amalgama, pigmentación fisiológica (11); también con lesiones de origen vascular como el Sarcoma de Kaposi y frente a una lesión de naturaleza posiblemente maligna y no pigmentada se debe plantear como posibles diagnósticos además del MOP el carcinoma oral de células escamosas. Aunque algunas de estas lesiones se diagnostican en base al historial médico y examen clínico; el examen histológico de todas las lesiones focales pigmentadas se impone para poder establecer un diagnóstico precoz y definitivo que abale un correcto y oportuno tratamiento (2). Tradicionalmente frente a una lesión pigmentada de la mucosa oral, se recomendaba la realización de una biopsia excéresis, con la finalidad de disminuir el riesgo de una posible diseminación/ siembra de células neoplásicas, actualmente este postulado carece de valor, es más, se recomienda biopsia incisional para toda lesión mayor a un centímetro, independientemente de su posible naturaleza histológica; permitiendo así, que fren- 
te a una neoplasia maligna se pueda planificar adecuadamente la cirugía oncológica con los debidos márgenes de seguridad (12).

Histopatológicamente, MOP puede presentar tres patrones: in situ (15\%) donde la neoplasia se encuentra limitada al epitelio y a la interface epitelio-conectiva (unión), invasivo o nodular (30\%) en el que se encuentra células neoplásicas proliferando en el tejido conjuntivo subepitelial y un patrón combinado (55\%) típico de la lesión avanzada, en el que se combina la proliferación neoplásica en la profundidad del tejido conjuntivo con actividad proliferativa maligna intraepitelial $(2,6,9)$.

Las células neoplásicas pueden exhibir morfología celular variada: fusiforme, epitelioide, plasmocitoide, o combinación de las anteriores, las que se organizan en distintos patrones: sólido, alveolar, organoide o pagetoide, con producción variable de melanina. También se pueden observar características adicionales de malignidad como ser necrosis, invasión perineural y perivascular. En las etapas tempranas, la proliferación de melanocitos malignos se encuentra restringida al epitelio (fase de crecimiento radial) que rápidamente progresa e invade el tejido conjuntivo subepitelial (conformando la fase de crecimiento vertical). La mayoría de los MOP en el momento del diagnóstico presentan un patrón de crecimiento vertical, invadiendo en profundidad el tejido conjuntivo (9). Otra característica histopatológica importante es la presencia o ausencia de pigmento melánico (5). El MOP en su variante amelánico es menos frecuente que la variante pigmentada. Autores como Speece et al. consideran que la ausencia de melanina se debe a que las células neoplásicas tienen un menor grado de diferenciación, lo que explicaría la incapacidad en la producción de la enzima tirosinasa, que inhabilitaría la producción de melanina (9).

Cuando la pigmentación de melanina está presente, el diagnóstico histopatológico es obvio. Sin embargo, cuando la lesión es amelánica, el diagnóstico diferencial implica un amplio espectro de neoplasias malignas, como ser: (a) Neoplasias malignas de células fusiformes (leiomiosarcomas, carcinoma de células fusiformes, tumor maligno de la vaina nerviosa periférica, sarcoma pleomórfico indiferenciado, angiosarcoma, sarcoma sinovial), (b) variantes epitelioides de tumores malignos (por ejemplo, sarcoma epitelioide, tumor maligno de la vaina nerviosa periférica de morfología epitelioide), (c) tumores de células azules y redondas (linfomas, tumores neuroendocrinos, rabdomiosarcoma, plasmacitoma o mieloma múltiple), (d) tumores malignos con células claras (carcinoma mucoepidermoide, carcinoma de células renales), y (e) variedad de lesiones metastásicas $(2,5)$. Por lo tanto, para poder establecer el diagnóstico de melanoma amelánico es fundamental el uso de inmunohistoquímica (2), siendo el panel sugerido, el constituido por: proteína S-100, tirosinasa, Melan-A y HMB-45 $(2,3,5)$. El MOP es negativo para citoqueratinas, EMA, desmina (2).

Debido a la rareza del MOP, para poder establecer su presencia, se debe cumplir con los criterios originalmente definidos por Greene et al. en 1953 a) actividad proliferativa neoplásica melanocítica en la unión epitelio-conectiva ( actividad de unión), b) evidencia clínica de melanoma maligno en la mucosa oral, y c) exclusión de otro posible sitio primario de origen. Cuando estos criterios no se cumplen, la lesión se considera metastásica $(1,5,9)$. El pronóstico del MOP es malo (7), debido principalmente, al diagnóstico inicial tardío $(2,3)$, la dificultad de alcanzar una resección amplia y a la tendencia de esta neoplasia de producir metástasis a distancia vía hematógena en forma muy temprana (9). Se estima que el $36 \%$ de los pacientes con MOP presentan compromiso ganglionar regional al momento del diagnóstico y el $85 \%$ de ellos desarrollan metástasis al hígado, pulmones, hueso o cerebro en un corto periodo de tiempo (6). La tasa de supervivencia a los 5 años para el MOP oscila en la literatura entre $15-25 \%$ hasta el $40 \%$ $(2,4,7,13)$, con una media de supervivencia menor de 2 años $(8,10)$. Entre los factores con valor 
pronóstico, se reconocen: edad avanzada, tamaño inicial de la lesión, enucleación quirúrgica deficiente, MOP amelánico y compromiso ganglionar regional en el momento del diagnóstico (2). También se asocian a un pobre diagnóstico el reconocimiento histológico de un índice mitótico elevado y la presencia de invasión vascular o neural $(2,11)$. La estadificación del MOP surge en paralelo con la utilizada para el melanoma cutáneo. Actualmente, se emplea la clasificación de la American Joint Comitee on Cancer (AJCC), quien no reconoce para el MOP los estadios T1 y T2 del sistema TNM, debido a su naturaleza agresiva e incidencia extremadamente baja; así los MOP se clasifican con un T3 (lesión delimitada al espesor de la mucosa oral), T4a (neoplasia moderadamente avanzada que compromete la profundidad de la mucosa oral, cartílago, hueso o piel) y T4b ( enfermedad muy avanzada, que involucra órganos distantes como cerebro, duramadre, base de cráneo, espacio masticador, arteria carótida, espacio prevertebral y mediastino) (7). Mientras que para el melanoma cutáneo,en base al conocimiento de las vías moleculares involucradas en la etiopatogenia, se encuentran perfectamente definidos los parámetros pronósticos, de estadificación y los blancos moleculares terapéuticos, no ocurre lo mismo para el MOP, cuya etiopatogenia es poco conocida, no pudiendo aún establecer blancos moleculares terapéuticos (14). En la patogénesis del melanoma cutáneo se ha demostrado la mutación de los oncogenes BRAF, NRAS y KIT, permitiendo el diseño de quimioterápicos que los tienen como blancos moleculares, así el uso terapéutico de Vemurafenib y el Dabrafenib (inhibidores selectivos de BRAF), se asocia con el aumento del período de sobrevida libre de enfermedad para el melanoma cutáneo, siendo su uso de rutina en la práctica clínica. Desafortunadamente los MOPs expresan en forma muy baja mutación de BRAF, NRAS y KIT (14).
Actualmente el tratamiento más efectivo para los MM es la resección quirúrgica con amplios márgenes de seguridad, complementada con la disección de cuello para metástasis de ganglios linfáticos positivos y en forma coadyuvante en pacientes de alto riesgo, sin estudios científicos que los avalen por completo se emplea quimioterapia, radioterapia e inmunoterapia $(1,2,7,9)$. Estas terapéuticas se consideran complementarias al tratamiento quirúrgico, debido a que ninguna de ellas ha aumentado de manera significativa el período de vida libre de cáncer post terapéutico, pero se continúan utilizando con el fin de poder brindar al paciente un eventual mejor pronóstico, ya que el MOP puede recurrir incluso aun cuando fue resecado completamente con grandes márgenes de seguridad (11).

\section{CONCLUSIONES}

EI MOP es un cáncer poco frecuente y agresivo, del que se desconoce en gran parte su etiopatogenia, y donde aún el tratamiento fundamental es el quirúrgico, el que resulta en la mayoría de los casos mutilante.

Es fundamental que los clínicos conozcan la existencia de esta entidad, de forma de propiciar, siempre que sea posible, un diagnóstico precoz que redunde en mejor y mayor sobrevida. Para poder detectar en forma temprana este tipo de cáncer resulta fundamental que el profesional conozca esta entidad, priorizando un examen detallado de la mucosa oral y la biopsia de toda lesión sospechosa nodular o macular de paladar o encía, independientemente del color y pigmentación.

DECLARACIÓN DE CONFLICTOS DE INTERESES: Los autores no reportan ningún conflicto de interés. El estudio se realizó con recursos propios de los autores y/o la institución a la que representan. 


\section{REFERENCIAS}

(1) Pingarrón Martín L, Martín-Moro JG, Ma CY, Yu ZW, Zhang CP. Melanoma de mucosa intraoral: ¿enfermedad local o sistémica?

Rev Esp Cir Oral y Maxilofac 2014; 36(1):1520. http://dx.doi.org/10.1016/j.maxilo.2012.07.001

(2) Deyhimi P, Razavi SM, Shahnaseri S, Khalesi S, HomayoniS, TavakoliP.Rareand ExtensiveMalignant Melanoma of the Oral Cavity: Report of Two Cases. J Dent (Shiraz, Iran) [Internet] 2017; 18(3):227-33. Available from: http://www.ncbi.nlm.nih.gov/pumed/29034279\%0Ahttp://www.pubmedcentral. nih.gov/articlerender.fcgi?artid=PMC5634364 [Consulta 20/02/2019].

(3) Maldonado-Mendoza J, Ramírez-Amador V, Anaya-Saavedra G, Irigoyen-Camacho ME, Ruíz-Godoy L, Ruíz-García E, et al. Clinicopathological characterization of primary oral and sinonasal melanoma in a referral centre in Mexico City: 20002012. Int J Oral Maxillofac Surg 2015; 44(4):427-32. doi: 10.1016/j.jom.2014.10.022

(4) Sortino-Rachou AM, Cancela M de C, Voti L, Curado MP. Primary oral melanoma: Populationbased incidence. Oral Oncol 2009; 45(3):254-8. http://dx.doi.org/10.1016/j.oraloncology.2008.04.015

(5) Smith MH, Bhattacharyya I, Cohen DM, Islam NM, Fitzpatrick SG, Montague LJ, et al. Melanoma of the Oral Cavity: an Analysis of 46 New Cases with Emphasis on Clinical and Histopathologic Characteristics.

Head Neck Pathol 2016; 10(3):298-305.

doi: 10.1007/s12105-016-0693-x

(6) Tlholoe MM, Khammissa RAG, Bouckaert M, Altini M, Lemmer J, Feller L. Oral Mucosal Melanoma: Some Pathobiological Considerations and an Illustrative Report of a Case.

Head Neck Pathol 2015; 9(1):127-34.

doi:10.1007/s12105-014-0526-8

(7) Wu Y, Wang L, Ma X, Guo W, Ren G. The existence of early stage oral mucosal melanoma:
A 10-year retrospective analysis of 170 patients in a single institute. Oral Oncol 2018; 87(October):70-6. https://doi.org/10.1016/j.oraloncology.2018.10.022

(8) Patel PB, Wright JM, Kang DR, Cheng YS. Longitudinal clinicopathologic data of the progression of oral mucosal melanoma - report of 2 cases and literature review. Oral Surg Oral Med Oral Pathol Oral Radiol [Internet]. 2018;126(1):e21-30. https://doi.org/10.1016/j.0ooo.2017.12.015

(9) de Castro MS, Pereira AAC, Sperandio FF, Reis BS de A, Nogueira DA, de Carli ML, et al. Primary oral melanoma: A clinicopathologic review and case presentation.

Quintessence Int (Berl) 2017; 48(10):815-27. doi: 10.3290/j.qi.a39077

(10) Mahmood H, Chengot P. Primary Malignant Gingival Melanoma: Case Report and Educational Reminder. Oral Surg 2017; 10(4):e88-91.

doi.org/10.1111/ors.12283

(11) Zapata S, Núñez L, Zamorano G, Villanueva J. Melanoma oral: Importancia de un diagnóstico temprano. Un caso clínico. Rev Clínica Periodoncia, Implantol y Rehabil Oral 2017; 10(2):90-2. http://dx.doi.org/10.4067/S0719-01072017000200090

(12) Kruse ALD, Riener MO, Graetz KW, Luebbers HT. Mucosal malignant melanomas in head and neck surgery: A retrospective study of six patients and review of the literature.

Oral Maxillofac Surg 2010; 14(3):143-7. doi: 10.1007/s10006-010-0207-z

(13) Singh H, Kumar P, Augustine J, Urs AB, Gupta S. Primary malignant melanoma of oral cavity: A report of three rare cases.

Contemp Clin Dent 2016; 7(1):87-9.

doi: 10.4103/0976-237X.177094

(14) Öztürk Sari Ş, Yilmaz Ii, Taşkin OÇ, Narli Gi, Şen F, Çomoğlu Ş, et al. BRAF, NRAS, KIT, TERT, GNAQ/GNA11 mutation profile analysis of head and neck mucosal melanomas: a study of 42 cases. Pathology 2017; 49(1):55-61. doi: 10.1016/j.pathol.2016.09.065 